Electronic version of an article published as [Lecture Notes in Control and Information Sciences, 2007, vol. 366, p. 157-169]

[DOI: http://dx.doi.org/10.1007/978-3-540-73890-9_12] @ [copyright Springer Verlag] [http://www.springerlink.com/content/b6054561l7t07111/] 


\title{
Simultaneous Interconnection and Damping Assignment Passivity-Based Control: Two Practical Examples
}

\author{
C. Batlle ${ }^{1}$, A. Dòria-Cerezo ${ }^{1}$, G. Espinosa-Pérez ${ }^{2}$, and R. Ortega ${ }^{3}$ \\ 1 MA4, DEE and IOC, EPSEVG, UPC, Av. V. Balaguer s/n, Vilanova i la \\ Geltrú, 08800, SPAIN, carles.batlle@upc.edu, arnau.doria@upc.edu \\ 2 DEPFI-UNAM, Apartado Postal 70-256, 04510 México D.F., MEXICO, \\ gerardoe@servidor. unam.mx \\ 3 Laboratoire des Signaux et Systémes, SUPELEC, Plateau du Moulon, \\ Gif-sur-Yvette 91192, FRANCE, Romeo.Ortega@lss.supelec.fr
}

\section{Introduction}

Passivity-based control (PBC) is a generic name given to a family of controller design techniques that achieves system stabilization via the route of passivation, that is, rendering the closed-loop system passive with a desired storage function (that usually qualifies as a Lyapunov function for the stability analysis.) If the passivity property turns out to be output strict, with an output signal with respect to which the system is detectable, then asymptotic stability is ensured. See the monographs $[5,12]$, and [6] for a recent survey.

As is well-known, [12], a passive system can be rendered strictly passive simply adding a negative feedback loop around the passive output - an action sometimes called $L_{g} V$ control, [10]. For this reason, it has been found convenient in some applications, in particular for mechanical systems, [11], [8], to split the control action into the sum of two terms, an energy-shaping term which, as indicated by its name, is responsible of assigning the desired energy/storage function to the passive map, and a second $L_{g} V$ term that injects damping for asymptotic stability. The purpose of this paper is to bring to the readers attention the fact that splitting the control action in this way is not without loss of generality, and effectively reduces the set of problems that can be solved via PBC. This assertion is, of course, not surprising since it is clear that, to achieve strict passivity, the procedure described above is just one of many other possible ways. Our point is illustrated with the IDA-PBC design methodology proposed in [4]. To enlarge the set of systems that can be stabilized via IDA-PBC we suggest to carry out simultaneously the energy shaping and the damping injection stages and refer to this variation of the method as SIDA-PBC. 
We illustrate the application of SIDA-PBC with two practically important examples. First, we show that the fundamental problem of induction motor torque and rotor flux regulation cannot be solved with two stage IDA-PBC. It is, however, solvable with SIDA-PBC. Second, we prove that with SIDA-PBC we can shape the total energy of the full (electrical and mechanical) dynamics of a doubly-fed induction generator used in power flow regulation tasks while, as reported in [1], with two stage IDA-PBC only the electrical energy could be shaped. Simulation results of these examples are presented to illustrate the performance improvement obtained with SIDA-PBC.

\section{PBC with Simultaneous Energy Shaping and Damping Injection}

We consider the problem of stabilization of an equilibrium point for nonlinear systems of the form

$$
\dot{x}=f(x, t)+g(x) u
$$

where $x \in \mathbb{R}^{n}$ is the state vector, $u \in \mathbb{R}^{m}, m<n$ is the control action and $g(x)$ is assumed full rank. In two-stage IDA-PBC this objective is achieved as follows, see $[4,8]$ for further details. First, decompose the control signal in two terms

$$
u=u_{e s}+u_{d i}
$$

where $u_{e s}$ is responsible of the energy-shaping stage and $u_{d i}$ injects the damping. Second, solve the key matching equation ${ }^{4}$

$$
g^{\perp}(x) f(x, t)=g^{\perp}(x) J_{d}(x, t) \nabla H_{d}
$$

for some functions

$$
J_{d}: \mathbb{R}^{n} \times \mathbb{R} \rightarrow \mathbb{R}^{n \times n}, \quad H_{d}: \mathbb{R}^{n} \rightarrow \mathbb{R},
$$

satisfying the skew-symmetry condition for the interconnection matrix

$$
J_{d}(x, t)+J_{d}^{\top}(x, t)=0
$$

and the equilibrium assignment condition for the desired total stored energy

$$
x_{\star}=\arg \min H_{d}(x)
$$

with $x_{\star} \in \mathbb{R}^{n}$ the equilibrium to be stabilized ${ }^{5}$ and $g^{\perp}(x) \in \mathbb{R}^{(n-m) \times n}$ a fullrank left-annihilator of $g(x)$, that is, $g^{\perp}(x) g(x)=0$ and rank $g^{\perp}(x)=n-m$.

As shown in [4] system (1) in closed-loop with the control (2), with

\footnotetext{
${ }^{4}$ All vectors in the paper are column vectors, even the gradient of a scalar function denoted $\nabla_{(\cdot)}=\frac{\partial}{\partial(\cdot)}$. When clear from the context the subindex will be omitted.

${ }^{5}$ That is, $x_{\star}$ is a member of the set $\left\{\bar{x} \in \mathbb{R}^{n} \mid g^{\perp}(\bar{x}) f(\bar{x}, t)=0, \forall t \in \mathbb{R}\right\}$.
} 


$$
u_{e s}=\left[g^{\top}(x) g(x)\right]^{-1} g^{\top}(x)\left\{J_{d}(x, t) \nabla H_{d}-f(x, t)\right\} .
$$

yields a port-controlled Hamiltonian $(\mathrm{PCH})$ system of the form

$$
\begin{aligned}
\dot{x} & =J_{d}(x, t) \nabla H_{d}+g(x) u_{d i} \\
y & =g^{\top}(x) \nabla H_{d} .
\end{aligned}
$$

The system (7) without damping injection term is conservative, i.e., $\dot{H}_{d}=0$, with $x_{\star}$ a stable equilibrium (with Lyapunov function $H_{d}(x)$ ). To add dissipation we feedback the passive output $y$, for instance, with

$$
u_{d i}=-K_{d i} y, K_{d i}=K_{d i}^{\top}>0,
$$

to finally obtain the PCH system with dissipation

$$
\begin{aligned}
\dot{x} & =\left[J_{d}(x, t)-R_{d}(x)\right] \nabla H_{d}+g(x) v \\
y & =g^{\top}(x) \nabla H_{d} .
\end{aligned}
$$

where the damping matrix $R_{d}(x)=R_{d}^{\top}(x) \geq 0$ is defined by

$$
R_{d}(x)=g(x) K_{d i} g^{\top}(x),
$$

and we have added a signal $v$ to $(2)$ to define the port variables. Since the new closed-loop system (with $v=0$ ) satisfies $\dot{H}_{d}=-y^{\top} K_{d i} y$, it can be proved (see for example Lemma 3.2.8 of [12] for the autonomous systems case) that the equilibrium $x_{\star}$ will now be asymptotically stable if it is detectable from $y$, i.e., if the implication $\left(y(t) \equiv 0 \Rightarrow \lim _{t \rightarrow \infty} x(t)=x_{\star}\right)$ is true.

Obviously, the key for the success of IDA-PBC is the solution of the matching equation (3). With the motivation of enlarging the class of systems for which this equation is solvable we propose in this paper to avoid the decomposition of the control into energy-shaping and damping injection terms. Instead, we suggest to carry out simultaneously both stages and replace (3), with the SIDA-PBC matching equations

$$
g^{\perp}(x) f(x, t)=g^{\perp}(x) F_{d}(x, t) \nabla H_{d},
$$

to replace the constraint (4) by the strictly weaker condition

$$
F_{d}(x, t)+F_{d}^{\top}(x, t) \leq 0,
$$

and define the control as

$$
u=\left[g^{\top}(x) g(x)\right]^{-1} g^{\top}(x)\left\{F_{d}(x, t) \nabla H_{d}-f(x, t)\right\} .
$$

Since the set of skew-symmetric matrices is strictly contained in the set of matrices with negative semi-definite symmetric part, it is clear that the set of functions $\{f(x, t), g(x)\}$ for which (3) - subject to the constraint (4)is solvable is strictly smaller than the set for which (9), subject to (10), is solvable. 
Remark 1. There exists several techniques to solve the matching equations (3) (resp., (9)), with two extremes cases being the purely algebraic approach of [2] and the PDE approach of [4]. In the former $H_{d}(x)$ is a priori fixed, which makes (3) (resp., (9)) an algebraic equation that is solved for $J_{d}(x, t)$ (resp., $F_{d}(x, t)$ ) - subject to the constraint (4) (resp., (10)). On the other hand, in the latter $J_{d}(x, t)$ (resp., $F_{d}(x, t)$ ) is fixed making (3) (resp., (9)) a PDE that is solved for $H_{d}(x)$. We refer the interested reader to [6] for a detailed discussion on these, as well as other, methods of solution of the matching equations. In this paper we will adopt the algebraic approach.

Remark 2. Similarly to IDA-PBC, application of SIDA-PBC also yields a closed-loop PCH system of the form (8) with

$$
\begin{aligned}
& J_{d}(x, t)=\frac{1}{2}\left[F_{d}(x, t)-F_{d}^{\top}(x, t)\right], \\
& R_{d}(x, t)=\frac{1}{2}\left[F_{d}(x, t)+F_{d}^{\top}(x, t)\right] .
\end{aligned}
$$

Remark 3. To make IDA-PBC applicable to non-autonomous systems, which will be required in the induction motor application, we have presented above a slight variation of the method. Notice that the matrices $J_{d}$ and $R_{d}$ may depend explicitly on time. Clearly, their skew-symmetry and non-negativity properties must now hold uniformly in time as well.

\section{Induction Motor Control via SIDA-PBC}

In this section we will show that the problem of output feedback torque control of induction motors is not solvable via two-stage IDA-PBC but it is solvable with SIDA-PBC. An interesting feature of our SIDA-PBC is that we establish here (Lyapunov) stability of a given equilibrium that generates the desired torque and rotor flux amplitude.

The standard two-phase model, which rotates at an arbitrary speed $\omega_{s} \in$ $\mathbb{R}$, is given by [13]

$$
\begin{aligned}
\dot{x}_{12}= & -\left[\gamma I_{2}+\left(n_{p} \omega+u_{3}\right) \mathcal{J}\right] x_{12} \\
& +\alpha_{1}\left(I_{2}-T_{r} n_{p} \omega \mathcal{J}\right) x_{34}+\alpha_{2} u_{12} \\
\dot{x}_{34}= & -\left(\frac{1}{T_{r}} I_{2}+\mathcal{J} u_{3}\right) x_{34}+\frac{L_{s r}}{T_{r}} x_{12} \\
\dot{\omega}= & \alpha_{3} x_{12}^{\top} \mathcal{J} x_{34}-\frac{\tau_{L}}{J_{m}}
\end{aligned}
$$

in which $I_{2} \in \mathbb{R}^{2 \times 2}$ is the identity matrix, $\mathcal{J}=-\mathcal{J}^{T} \in \mathbb{R}^{2 \times 2}, x_{12} \in \mathbb{R}^{2}$ are the stator currents, $x_{34} \in \mathbb{R}^{2}$ the rotor fluxes, $\omega \in \mathbb{R}$ the rotor speed, $u_{12} \in \mathbb{R}^{2}$ are the stator voltages, $\tau_{L} \in \mathbb{R}$ is the load torque and $u_{3}:=\omega_{s}-n_{p} \omega$. All the parameters are positive and defined in the usual way. Notice that, as first pointed out in the control literature in [7], the signal $u_{3}$ acts as an additional 
control input. We will select $u_{3}$ to transform the periodic orbits of the system into constant equilibria.

We are interested in this paper in the problem of regulation of the motor torque and the rotor flux amplitude

$$
y=\left[\begin{array}{l}
y_{1} \\
y_{2}
\end{array}\right]=\left[\begin{array}{c}
J_{m} \alpha_{3} x_{12}^{\top} \mathcal{J} x_{34} \\
\left|x_{34}\right|
\end{array}\right],
$$

to some constant desired values $y_{\star}=\operatorname{col}\left(y_{1 \star}, y_{2 \star}\right)$, where $|\cdot|$ is the Euclidean norm, assuming that the only signals available for measurement are $x_{12}$ and $\omega$.

To solve this problem using (S)IDA-PBC it is necessary to express the control objective in terms of a desired equilibrium. In this sense, it can be shown that for the induction motor model (11)-(13) with output functions (14) and

$$
u_{3}=u_{3 \star}:=\frac{R_{r}}{n_{p}} \frac{y_{1 \star}}{y_{2 \star}^{2}} .
$$

the set of assignable equilibrium points, denoted $\operatorname{col}\left(\bar{x}_{12}, \bar{x}_{34}, \bar{\omega}\right) \in \mathbb{R}^{5}$, which are compatible with the desired outputs $y_{\star}$ is defined by

$$
\begin{aligned}
\bar{x}_{12} & =\frac{1}{L_{s r}}\left[\begin{array}{cc}
1 & -\frac{L_{r}}{n_{p}} \frac{y_{1 \star}}{y_{2 \star}^{2}} \\
\frac{L_{r}}{n_{p}} \frac{y_{1 \star}}{y_{2 \star}^{2}} & 1
\end{array}\right] \bar{x}_{34} \\
\left|\bar{x}_{34}\right| & =y_{2 \star}
\end{aligned}
$$

with $\bar{\omega}$ arbitrary.

Remark 4. From (13) and (14) we see that to operate the system in equilibrium, $y_{1 \star}=\tau_{L}$-hence, to define the desired equilibrium the load torque needs to be known. In practical applications, an outer loop PI control around the velocity error is usually added. The output of the integrator, on one hand, provides an estimate of $\tau_{L}$ while, on the other hand, ensures that speed also converges to the desired value as shown via simulations below. A scheme that removes this assumption has recently been proposed in [3].

As indicated in Remark 1, in this paper we will adopt the algebraic approach to solve the matching equations. To this end, we will consider a quadratic in errors energy function of the form

$$
H_{d}(x)=\frac{1}{2}\left(x-x_{\star}\right)^{\top} P\left(x-x_{\star}\right),
$$

with $P=P^{\top}>0$ a matrix to be determined. Moreover, the problem formulation is simplified by using the generic symbol $F(x, t)$ to denote either $J_{d}(x, t)$ or $F_{d}(x, t)$, and identifying the IDA and SIDA approaches by imposing either $F(x, t)+F^{\top}(x, t)=0$ or $F(x, t)+F^{\top}(x, t) \leq 0$, respectively. 
Since we are interested here in torque control, and this is only defined by the stator currents and the rotor fluxes, its regulation can be achieved applying IDA-PBC to the electrical subsystem only. Boundedness of $\omega$ will be established in a subsequent analysis.

If the electrical subsystem (11), (12), with $u_{3}=u_{3 \star}$ and $u=u_{12}$, is written in the form (1), then, selecting $g^{\perp}=\left[0_{2 \times 2} I_{2}\right]$, it is possible to notice that the matching equations (3) and (9) concern only the third and fourth rows of $f(x, t)$ and they take the form

$$
-\left(\frac{1}{T_{r}} I_{2}+\mathcal{J} u_{3 \star}\right) x_{34}+\frac{L_{s r}}{T_{r}} x_{12}=\left[\begin{array}{ll}
F_{3}(x, t) & \left.F_{4}(x, t)\right] P\left(x-x_{\star}\right),
\end{array}\right.
$$

where, to simplify the notation, we partition $F(x, t)$ into $F_{i} \in \mathbb{R}^{2 \times 2}, i=$ $1, \ldots, 4$, sub-matrices.

On the other hand, considering the constraint imposed by the possibility of measuring only $x_{12}$ and $\omega$, from (6) it is possible to see that the control can be written, factoring the components that depend on the unmeasurable quantity $x_{34}$, as

$$
u_{12}=\hat{u}_{12}\left(x_{12}, \omega\right)+\frac{1}{\alpha_{2}} S(x, t) x_{34}
$$

with $\hat{u}_{12}\left(x_{12}, \omega\right)$ given in $(21)$. Hence, it is clear that to verify the output feedback condition it must be satisfied that

$$
S(x, t):=\alpha_{1}\left[T_{r} n_{p} \omega(t) \mathcal{J}-I_{2}\right]+F_{1}(x, t) P_{2}+F_{2}(x, t) P_{3}=0,
$$

where we have partitioned the symmetric matrix $P$ into $P_{i} \in \mathbb{R}^{2 \times 2}, i=1,2,3$, sub-matrices.

In order to show that the energy-shaping problem is not solvable, it is possible to establish that condition $F(x, t)+F^{\top}(x, t)=0$ is equivalent to

$$
G=P_{3}^{-1}\left(-G^{\top}\right) P_{3}
$$

where

$$
G:=\frac{1}{\lambda}\left[\frac{L_{s r}}{T_{r}} P_{3}+P_{1}\left(\alpha_{1} I_{2}-\lambda \beta_{2} \mathcal{J}\right)\right]
$$

with $\lambda \in \mathbb{R}, \lambda \neq 0, \beta_{2} \in \mathbb{R}$ and $P_{3}$ a full rank matrix.

Consequently, $G$ must be similar to $-G^{\top}$, and both necessarily have the same eigenvalues. A necessary condition for the latter is that $\operatorname{trace}(G)=0$, that clearly is not satisfied.

On the other hand, it can be shown that $F_{2}(t)=\alpha_{1}\left[I_{2}-T_{r} n_{p} \omega(t) \mathcal{J}\right] P_{3}^{-1}$, $F_{3}=\frac{L_{s r}}{T_{r}} P_{1}^{-1}, F_{4}=-\left(\frac{1}{T_{r}} I_{2}+u_{3 \star} \mathcal{J}\right) P_{3}^{-1}$ and $P_{2}=0$, with $F_{1}(x, t), P_{1}, P_{3}$ free, provide a solution to (18) and (19). Moreover, if $P_{1}=\frac{L_{s r}}{T_{r}} I_{2}, P_{3}=\alpha_{1} I_{2}$ and $F_{1}(t)=-\mathcal{K}(\omega(t))$, with

$$
\mathcal{K}(\omega(t))>\frac{L_{s r}}{L_{s} L_{r}-L_{s r}^{2}}\left[1+\frac{1}{4}\left(T_{r} n_{p} \omega(t)\right)^{2}\right] I_{2}
$$


then $F(t)+F^{\top}(t)<0$.

The final part of the design is the explicit definition of the resulting controller, given by $u_{3}=\frac{R_{r}}{n_{p}} \frac{y_{1 \star}}{y_{2 \star}^{2}}$ and $u_{12}=\hat{u}_{12}\left(x_{12}, \omega\right)$ with

$$
\begin{aligned}
\hat{u}_{12}\left(x_{12}, \omega\right)= & \frac{1}{\alpha_{2}}\left[\gamma I_{2}+\left(n_{p} \omega+u_{3 \star}\right) \mathcal{J}\right] x_{12}-\frac{\alpha_{1}}{\alpha_{2}}\left(I_{2}-T_{r} n_{p} \omega \mathcal{J}\right) x_{34 \star} \\
& -\frac{L_{s r}}{\alpha_{2} T_{r}} \mathcal{K}(\omega)\left(x_{12}-x_{12 \star}\right)
\end{aligned}
$$

and $\mathcal{K}(\omega)$ satisfying (20), which guarantees that the equilibrium $x_{\star}$ is globally exponentially stable while $\omega$ remains bounded.

The performance of the proposed SIDA-PBC was investigated by simulations using the motor parameters reported in [7]. The rotor flux equilibrium value was set to $x_{34 \star}=\operatorname{col}(\beta, 0)$ with $\beta=2$, while $x_{12 \star}$ were computed according to (16). In the experiment, with the motor at standstill and a startup zero load torque, a profile for this latter variable was considered going first to $\tau_{L}=20 \mathrm{Nm}$ and later on to $\tau_{L}=40 \mathrm{Nm}$. In Figure 1 it is shown how the generated torque regulation objective is achieved.

The second experiment was aimed to illustrate the claim stated in Remark 4 regarding the estimation of the load torque with speed control purposes. In this sense, the control input $u_{3}$ was set to

$$
u_{3}=\hat{u}_{3 \star}=R_{r} \frac{\hat{y}_{1 \star}}{y_{2 \star}^{2}}
$$

where the estimate of the load torque is obtained as the output of a PI controller, defined over the speed error between the actual and the desired velocities, of the form

$$
\hat{y}_{1 \star}(t)=k_{p}\left(\omega(t)-\omega_{\star}\right)+k_{i} \int_{0}^{t}\left(\omega(s)-\omega_{\star}\right) d s
$$

Figure 2 shows the rotor speed behavior when the desired velocity is initially $\omega_{\star}=100 \mathrm{rpm}$ and at $t=50 \mathrm{sec}$ it is changed to $\omega_{\star}=150 \mathrm{rpm}$. In this simulation it was considered $\tau_{L}=10 \mathrm{Nm}, k_{i}=-.1$ and $k_{p}=-1$. All the other parameters were the same than in the first experiment.

\section{Total Energy-Shaping of a Doubly-Fed Induction Generator}

The second considered example in this paper is related with the control of a doubly-fed induction machine (DFIM) [9]. In this case the device acts as an energy-switching device between a local energy storing element (a flywheel) and the electrical power network. The control objective is to change the direction of the power flow (towards or from the flywheel) depending on the load 


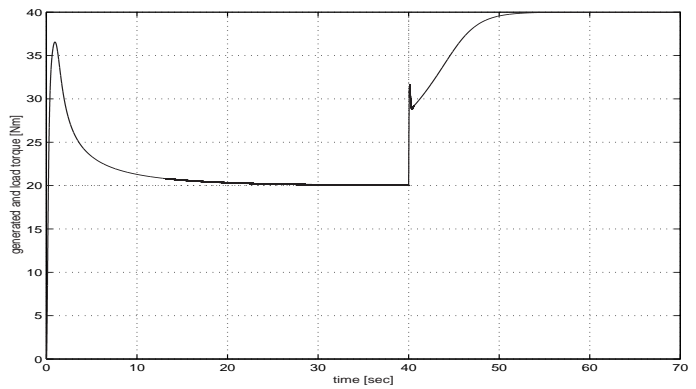

Fig. 1. Generated and load torque of the IM

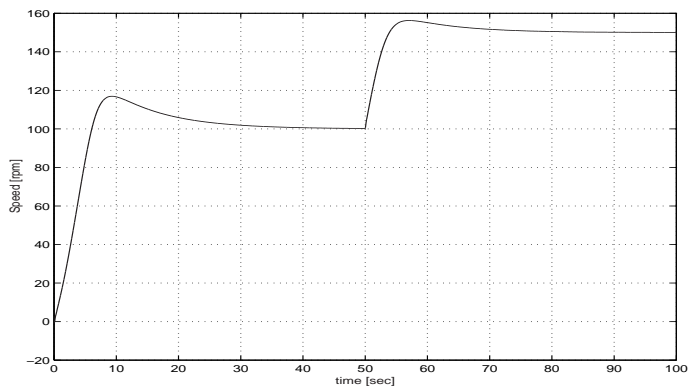

Fig. 2. Speed behavior with estimated load torque

demand. In [1] the equilibria associated to these regimes is stabilized with an IDA-PBC that shapes the electrical energy, treating the mechanical dynamics as a cascaded subsystem. The purpose of this section is to show that using SIDA-PBC it is possible to shape the energy function of the complete system dynamics, resulting in a controller with improved power-flow regulation performance due to the possibility of considering a fast response of the mechanical speed. To the best of our knowledge, this is the first control algorithm for this class of systems that provides for this additional degree of freedom.

We consider the configuration for the DFIM studied in [1] where a representation in the $d q$ framework rotating at the (constant) angular speed of the AC source $\left(\omega_{s}\right)$ is assumed. The energy function of the overall system is $H(z)=\frac{1}{2} z^{\top} \mathcal{L}^{-1} z$, and the model is given by

$$
\dot{z}=\left[J\left(i_{s}, \omega\right)-R\right] \nabla H+\left[\begin{array}{c}
v_{s} \\
O_{2 \times 1} \\
\tau_{L}
\end{array}\right]+\left[\begin{array}{c}
O_{2 \times 2} \\
I_{2} \\
O_{1 \times 2}
\end{array}\right] u
$$

where

$$
\mathcal{L}=\left[\begin{array}{ccc}
L_{s} I_{2} & L_{s r} I_{2} & O_{2 \times 1} \\
L_{s r} I_{2} & L_{r} I_{2} & O_{2 \times 1} \\
O_{1 \times 2} & O_{1 \times 2} & J_{m}
\end{array}\right]=\mathcal{L}^{\top}>0
$$


is the generalized inductance matrix, $v_{s} \in \mathbb{R}^{2}, \tau_{L} \in \mathbb{R}$ are, respectively, the stator voltage and the external mechanical torque, which are constant, $u \in \mathbb{R}^{2}$ are the rotor control voltages, $z=\operatorname{col}\left(\lambda_{s}, \lambda_{r}, J_{m} \omega\right) \in \mathbb{R}^{5}$, where $\lambda_{s}, \lambda_{r} \in \mathbb{R}^{2}$ are the stator and rotor fluxes, respectively, and $\omega \in \mathbb{R}$ is the mechanical speed. The (skew-symmetric) structure and damping matrices are

$$
\begin{gathered}
J\left(i_{s}, \omega\right):=\left[\begin{array}{ccc}
-\omega_{s} L_{s} \mathcal{J} & -\omega_{s} L_{s r} \mathcal{J} & O_{2 \times 1} \\
-\omega_{s} L_{s r} \mathcal{J} & -\left(\omega_{s}-\omega\right) L_{r} \mathcal{J} & L_{s r} \mathcal{J} i_{s} \\
O_{1 \times 2} & L_{s r} i_{s}^{\top} \mathcal{J} & 0
\end{array}\right], \\
R:=\left[\begin{array}{ccc}
R_{s} I_{2} & O_{2 \times 2} & O_{2 \times 1} \\
O_{2 \times 2} & R_{r} I_{2} & O_{2 \times 1} \\
O_{1 \times 2} & O_{1 \times 2} & B_{r}
\end{array}\right]>0
\end{gathered}
$$

All machine parameters are defined in the usual way. Also note that the vector $\chi=\operatorname{col}\left(i_{s}, i_{r}, \omega\right) \in \mathbb{R}^{5 \times 5}$, where $i_{s}, i_{r} \in \mathbb{R}^{2}$ are the stator and rotor currents, respectively, satisfies $z=\mathcal{L} \chi$.

Clearly, the fixed point equations for $(22)$ are given by $z_{\star}=\mathcal{L} \chi_{\star}$, with $\chi_{\star}:=\operatorname{col}\left(i_{s \star}, i_{r \star}, \omega_{\star}\right)$ the solutions of

$$
\begin{array}{r}
-\left(\omega_{s} L_{s} \mathcal{J}+R_{s} I_{2}\right) i_{s \star}-\omega_{s} L_{s r} \mathcal{J} i_{r \star}+v_{s}=0 \\
L_{s r} i_{s \star}^{\top} \mathcal{J} i_{r \star}-B_{r} \omega_{\star}+\tau_{L}=0,
\end{array}
$$

As discussed in [1], the direction of the power flow can be regulated commuting between two controllers that stabilize two different equilibrium points.

In order to obtain the controller that stabilizes the desired equilibrium, as done in Section 3 we will design our SIDA-PBC adopting the algebraic approach. For, we fix the desired energy function as

$$
H_{d}(z)=\frac{1}{2}\left(z-z_{\star}\right)^{\top} P\left(z-z_{\star}\right), P=P^{\top}>0 .
$$

Thus SIDA-PBC design reduces to finding a matrix $F_{d}(z)$ such that the righthand term of $(22)$ equals $F_{d}(z) P\left(z-z_{\star}\right)$ and verifying $F_{d}(z)+F_{d}^{\top}(z) \leq 0$. To simplify the solution we restrict $P$ to be diagonal while

$$
F_{d}(z)=\left[\begin{array}{ccc}
F_{11}(z) & F_{12}(z) & O_{2 \times 1} \\
F_{21}(z) & F_{22}(z) & F_{23}(z) \\
F_{31}^{\top}(z) & F_{32}^{\top}(z) & F_{33}(z)
\end{array}\right]
$$

It can be shown that if $F_{11}=-\frac{1}{p_{s}}\left(\omega_{s} \mathcal{J}+\frac{L_{r}}{\mu} R_{s} I_{2}\right)$ and $F_{12}=\frac{L_{s r}}{p_{r} \mu} R_{s} I_{2}$ with $\mu:=L_{s} L_{r}-L_{s r}^{2}>0$, with $F_{31}=\frac{L_{s r}}{p_{s} \mu} \mathcal{J} \lambda_{r \star}, F_{32}(z)=-\frac{L_{s r}}{p_{r} \mu} \mathcal{J} \lambda_{s}, F_{33}=-\frac{B_{r}}{p_{\omega} J_{m}}$, while

$$
F_{21}=-F_{12}, F_{23}(z)=-F_{32}(z), F_{22}=-\frac{k_{r}}{2 p_{r}} I_{2}<0
$$

yields 


$$
F_{d}(z)+F_{d}^{\top}(z)=\left[\begin{array}{ccc}
-\frac{2 L_{r} R_{s}}{p_{s} \mu} I_{2} & O_{2 \times 2} & \frac{L_{s r}}{p_{s} \mu} \mathcal{J} \lambda_{r \star} \\
O_{2 \times 2} & -\frac{k_{r}}{p_{r}} I_{2} & O_{2 \times 1} \\
-\frac{L_{s r}}{p_{s} \mu} \lambda_{r \star}^{\top} \mathcal{J} & O_{1 \times 2} & -\frac{2 B_{r}}{p_{\omega} J_{m}}
\end{array}\right] .
$$

A simple Schur's complement analysis establishes that $F_{d}(z)+F_{d}^{\top}(z)<0$ if and only if the free parameters $p_{s}$ and $p_{\omega}$ satisfy

$$
p_{s}>\left(\frac{J_{m} L_{s r}^{2}}{4 B_{r} L_{r} R_{s} \mu}\left|\lambda_{r \star}\right|^{2}\right) p_{\omega} .
$$

Once we have solved the SIDA-PBC matching equations, the design is completed computing the controller which in this case is given as the static feedback control

$$
\begin{aligned}
u & =R_{r} i_{r}+\left(\omega_{s}-\omega\right) \mathcal{J}\left(L_{s r} i_{s}+L_{r} i_{r}\right) \\
& -k_{s}\left(L_{s} \tilde{i}_{s}+L_{s r} \tilde{i}_{r}\right)-k_{r}\left(L_{s r} \tilde{i}_{s}+L_{r} \tilde{i}_{r}\right)+k_{\omega} \mathcal{J} \lambda_{s} \tilde{\omega}
\end{aligned}
$$

where $k_{r}>0, k_{\omega}>0$ and $k_{s}>\frac{L_{s r}^{2}}{4 B_{r} L_{r} \mu}\left|\lambda_{r \star}\right|^{2} k_{\omega}$. Considering this control law, the equilibrium $z_{\star}$ is globally exponentially stable.

In spite of the remarkable stability properties of the proposed scheme, it can be seen that (27) has an strong dependence on $R_{r}$, which is in general an uncertain parameter. With the aim of robustifying this control law it is possible to develope an adaptive version in the following way.

Replacing (27) by

$$
\begin{aligned}
u & =\left(\omega_{s}-\omega\right) \mathcal{J}\left(L_{s r} i_{s}+L_{r} i_{r}\right) \\
& -k_{s}\left(L_{s} \tilde{i}_{s}+L_{s r} \tilde{i}_{r}\right)-k_{r}\left(L_{s r} \tilde{i}_{s}+L_{r} \tilde{i}_{r}\right)+k_{\omega} \mathcal{J} \lambda_{s} \tilde{\omega}+\hat{R}_{r} i_{r},
\end{aligned}
$$

where $\hat{R}_{r}$ is an estimate of $R_{r}$ that we have to generate on-line, we define the parameter error $\tilde{R}_{r}=\hat{R}_{r}-R_{r}$. The closed-loop system has the form

$$
\dot{z}=F \nabla H_{d}+\tilde{R}_{r} B i_{r},
$$

where $B=\left[O_{2 \times 2}, I_{2}, O_{1 \times 2}\right]^{T}$.

To complete the design we propose a Lyapunov function

$$
W\left(z, \tilde{R}_{r}\right)=H_{d}+\frac{1}{2 k_{a}} \tilde{R}_{r}^{2}
$$

where $k_{a}>0$ is the adaptation gain. Its derivative yields, selecting $\dot{\tilde{R}}_{r}=$ $-k_{a}\left(\nabla H_{d}\right)^{\top} B i_{r}$, that

$$
\dot{W}=-\left(\nabla H_{d}\right)^{\top} F \nabla H_{d} \leq 0,
$$

which proves the stability of the adaptive system. The resulting adaptation law is 


$$
\dot{\tilde{R}}_{r}=-k_{a}\left(L_{s r}{\tilde{i_{s}}}^{\top}-L_{r}{\tilde{i_{r}}}^{\top}\right) p_{r} i_{r}
$$

The usefulness of the controller (27) was illustrated by some simulations using the DFIM parameters of [1]. The controller parameters were selected as $k_{s}=1000, k_{r}=100$ and $k_{\omega}=0.01$. In Figure 3 we compare the speed behavior of the new SIDA-PBC with the IDA-PBC reported in [1] - that shapes only the electrical energy. As can be noticed, SIDA-PBC achieves a much faster speed response.

To evaluate the adaptive controller (28) the same experiment as before was repeated but now varying the rotor resistance parameter $R_{r}$. At $t=0.5 \mathrm{~s}$ the value of $R_{r}$ of the model is smoothly increased to $R_{r}=0.02$, simulating temperature effects. Figures 4 and 5 show the estimation behavior of $\hat{R}_{r}$ and the dynamics of the mechanical speed, respectively.The convergence of the estimated value of $\hat{R}_{r}$ to the real value (Figure 4 ) ensures that the performance of the ideal system is recovered (Figure 5).
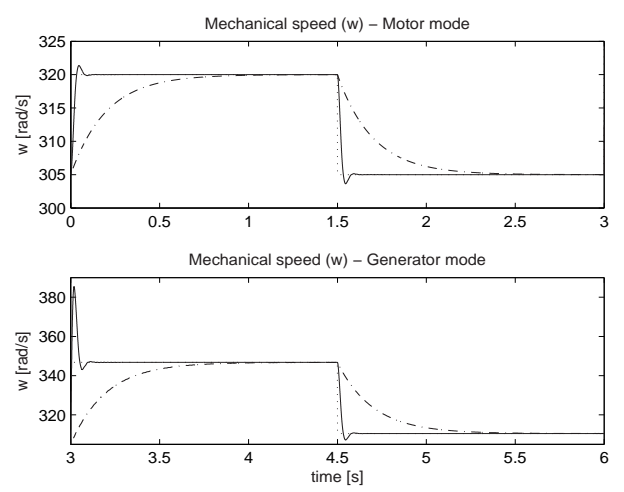

Fig. 3. Mechanical speed, $\omega$, for SIDA-PBC (continuous line) and IDA-PBC (dashed line).

\section{Conclusions}

We have presented an extension of the highly successful IDA-PBC methodology, called SIDA-PBC, where the energy-shaping and damping injection tasks are not performed sequentially, but simultaneously. In this way we enlarge the class of systems that can be stabilized using PBC and, furthermore, through the consideration of a broader set of desired damping matrices, we provide the designer with more tuning knobs to improve performance.

This new idea has been applied to solve the long standing problem of IDA-PBC of induction motors, that turns out to be unsolvable with a two 


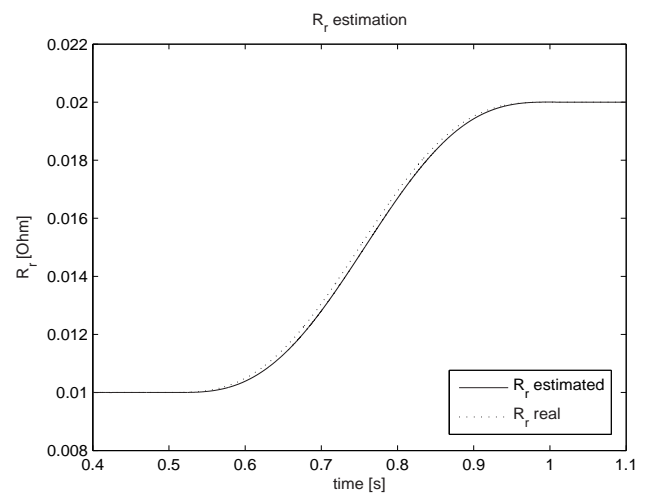

Fig. 4. Rotor resistance estimation, $\hat{R}_{r}$.

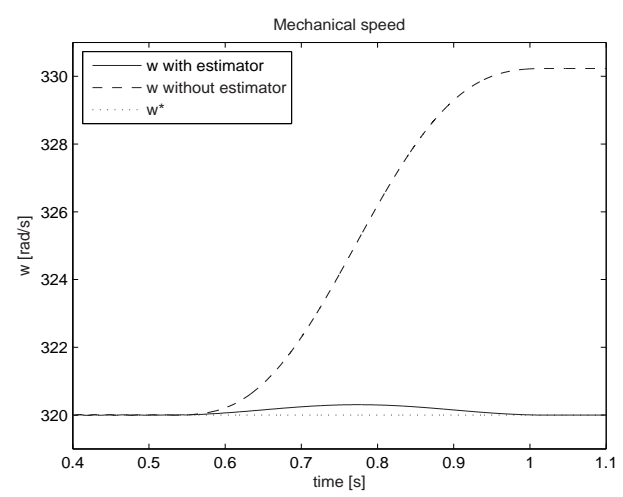

Fig. 5. Mechanical speed, with and without estimator.

stage design. Also, by avoiding the classical nested-loop control configuration prevalent in electromechanical systems, we have been able to improve the mechanical response of a DFIM, working both as a motor and a generator. Experimental validation of the two control algorithms is currently being terminated and will be reported in the near future.

\section{Acknowledgments}

This work has been done in the context of the European sponsored project Geoplex with reference code IST-2001-34166. Further information is available at http://www.geoplex.cc. The work of Carles Batlle, Arnau Dòria-Cerezo and Gerardo Espinosa has been (partially) supported by the spanish project DPI2004-06871-CO2-02, the European Community Marie Curie Fellowship 
(in the framework of the European Control Training Site) and CONACyT (51050Y) and DGAPA-UNAM (IN103306), respectively.

\section{References}

1. Batlle, C., A. Dòria-Cerezo and R. Ortega (2005) Power flow control of a doubly-fed induction machine coupled to a flywheel European Journal of Control 11:3 209-221

2. Fujimoto, K. and T. Sugie (2001) Canonical transformations and stabilization of generalized hamiltonian systems Systems and Control Letters 42:3 217-227

3. Karagiannis, D., A. Astolfi, R. Ortega and M. Hilairet (2005) A nonlinear tracking controller for voltage-fed induction motors with uncertain load torque. Internal report, LSS Supelec, France

4. Ortega, R., A. van der Schaft, B. Maschke and G. Escobar (2002) Interconnection and Damping Assignment Passivity-based Control of Port-controlled Hamiltonian Systems AUTOMATICA 38:4 585-596

5. Ortega, R., A. Loria, P.J. Nicklasson and H. Sira-Ramírez (1998) Passivitybased Control of Euler-Lagrange Systems. Communications and Control Engineering. Springer-Verlag, Berlin

6. Ortega, R. and E. Garcia-Canseco (2004) Interconnection and Damping Assignment Passivity-Based Control: A Survey European Journal of Control 10:432450

7. Ortega, R. and G. Espinosa (1993) Torque regulation of induction motors AUTOMATICA 47:8 621-633

8. Ortega, R., M. Spong, F. Gomez and G. Blankenstein (2002a) Stabilization of underactuated mechanical systems via interconnection and damping assignment IEEE Trans. Automatic Control 47:8 1218-1233

9. Peresada, S., A. Tilli and A. Tonelli (2004) Power control of a doubly fed induction machine via output feedback Control Engineering Practice 12:41-57

10. Sepulchre, R., M. Janković and P. Kokotović (1997) Constructive Nonlinear Control. Springer-Verlag, London

11. Takegaki, M. and S. Arimoto (1981) A new feedback for dynamic control of manipulators Trans. of the ASME: Journal of Dynamic Systems, Measurement and Control 102:119-125

12. van der Schaft, A. (2000) $L_{2}$-Gain and Passivity Techniques in Nonlinear Control. 2nd edition. Springer-Verlag, London

13. Krause, P.C., O. Wasynczuk and S.D. Sudhoff (1995) Analysis of Electric Machinery. IEEE Press, USA. 